\title{
NIP IT IN THE BUD - A POPULATION-BASED BREAST CANCER SURVEILLANCE PROGRAM
}

\section{Smitha Thomas Kaniyampady \\ Dr Srujan Goud Janagam*}

Deputy Manager CSR-Narayana Health.

\author{
Deputy Manager, CSR-Narayana Health. *Corresponding Author
}

\section{Dr. Sakshi Thakral Manager CSR Narayana Health.}

ABSTRACT Breast Cancer is the most common type of cancer among women. In addition to the burden of mortality and morbidity, Breast Cancer treatment cost and expenditure comprises a major chunk of the catastrophic health expenditure and financial burden incurred by the population. Delayed hospital presentation and diagnosis augments the hardships faced by individuals, family, community, and the health system at large. The need and urgency to create awareness about the disease and enable early detection via community level population-based screening was identified early in 2014. Through the awareness sessions, the program aims to demystify Breast Cancer and break the stigma associated with it. The intervention adopted a screening methodology that was cost effective and combination of Clinical Breast Examination and Mammography was followed. This paper discusses the learnings and outcomes of the surveillance over the years across geographies spreading awareness.

\section{KEYWORDS : Breast Cancer Screening, Population surveillance, Early detection, Low resource screening}

\section{INTRODUCTION}

Breast Cancer is a devastating disease whose incidence is drastically increasing globally. Cancer is an abnormal cell growth in a particular organ/region of the body with the potential to invade or spread to other parts of the body. Breast cancer can be simply defined as the cancer of cells in the breast. It usually manifests as a breast lump or thickening that feels different from the surrounding tissue, change in the size, shape or appearance of a breast, changes to the skin over the breast, such as dimpling, a newly inverted nipple, peeling, scaling, crusting or flaking of the pigmented area of skin surrounding the nipple (areola) or breast skin or redness and pitting of the breast skin.

A combination of factors contributes to risk for breast cancer. Although, some women will get breast cancer even without any other risk factors that they know of and, having a risk factor does not mean you will get the disease. Major risk factors include age, family history, genetics, exposure to radiation, ethnicity amongst others.

\section{Epidemiology of Breast Cancer in India}

Despite breast cancer having a high incidence there is a shortfall on reliable data sources and comprehensive studies pertaining to the diseases. As part of cancer surveillance, the National Cancer Registry Program was initiated in 1964 and expanded in 1982. There are currently 26 Population Based Registries and 7 Hospital Based Registries in India.

Twenty five percent of cancer cases reported amongst women are breast cancer. The rate of incidence was found to be 25.8 in 100,000 women and the mortality rate is 12.7 per 100,000 women (2017). The highest rate of occurrence was found to be in Delhi (4l per 100,000 women) followed by Chennai (37.9 per 100,000 women), Bangalore (34.4 per 100,000 women), and Thiruvananthapuram district (33.7 per 100,000 women). The mortality-to-incidence ratio was found to reach 0.66 in rural registries and 0.08 in urban registries.

Increased incidence of the disease in younger Indian women (between the ages of 30 and 40) was also observed. Almost $48 \%$ of patients with breast cancer in India are below 50 years of age. There is an increasing trend of breast cancer in women between the ages of 25 and 40 in the past 25 years.
Surveillance trends from 2000 to 2014 based on registries from 71 countries estimated the 5 -year survival ratio to be $66.1 \%$ in India which is the lowest levels among the countries included in their study.

\section{The Problem}

Breast cancer incidence is on the rise owing to urbanization and changes in lifestyle. It is a significant determinant of mortality and morbidity amongst women. Breast cancer cannot be prevented entirely, however, it can be treated more effectively and cured if detected at an earlier stage.

There are several factors that affect screening and early detection of breast cancer:

1. Awareness about breast cancer

2. Availability of screening facilities

3. Affordability for screening facilities

4. Stigma associated with breast cancer

5. Myths associated with breast cancer

\section{Materials and Methods}

Population based screening for early detection

The World Health Organization (WHO) defines screening as the presumptive identification of unrecognised disease or defects by means of tests, examinations or other procedures that can be applied rapidly. Screening is intended for all people, in an identified target population, who do not have symptoms of the disease or condition being screened for. The objective of screening is to reduce the burden due to the disease including the incidence, morbidity and mortality.

Screening can reduce the risk of developing or dying from a disease, but it does not guarantee that the disease will not occur or, if it occurs, that it can be cured. A 'positive' screening test identifies people who are at increased likelihood of having the condition and who require further investigation to determine whether they have the disease or condition.

In 1968, Wilson and Jungner developed the WHO principles of screening. These principles are outlined below. These principles remain relevant today when developing criteria for a specific country or screening issue. 


\section{WHO Principles of Early Disease Detection}

\section{Condition}

- The condition should be an important health problem.

- There should be a recognisable latent or early symptomatic stage.

- The natural history of the condition, including development from latent to declared disease, should be adequately understood.

\section{Test}

- There should be a suitable test or examination.

- The test should be acceptable to the population.

\section{Treatment}

- There should be an accepted treatment for patients with recognised disease.

\section{Screening Program}

- There should be an agreed policy on whom to treat as patients.

- Facilities for diagnosis and treatment should be available.

- The cost of case-findings (including diagnosis and treatment of patients diagnosed) should be economically balanced in relation to possible expenditure on medical care.

- Case-findings should be a continuing process and not a 'once and for all' project.

Breast Cancer qualifies as a disease that required population -based screening as per the Wilson and Jungner Criteria, hence this was taken up by Narayana Health CSR as a measure in fighting the burden due to the disease. A framework was developed based on which the intervention was structured.

\section{Theory of change and result framework}

The framework looks at reducing the mortality and morbidity of breast cancer through early detection. A single intervention cannot address every determinant hence a multi-level approach is adopted. A nuanced multi-level approach would go beyond a clinical approach and address the socio-culturaleconomic issues determining health.

Our program was influenced by two approaches, the Health Belief Model and the Systems approach. The Health Belief Model is a theory that enables better understanding of the individual determinants and the social determinants that influence the individual determinants. The Systems Approach is a broader model that looks at the larger ecosystem within which the various determinants exist in parallel. The determinants were predominantly identified to be individual, social and health system. The systems approach takes into consideration all the identified determinants and their relationship with each other while the health belief theory offers insight on the factors underlying individual and social determinants.

Shown below is the conceptual framework for the program based on the models discussed:

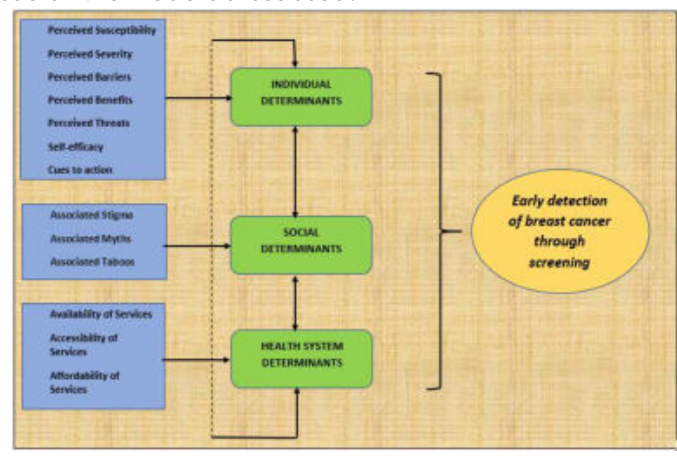

From existing literature and research, it is understood that early detection of breast cancer can help reduce the mortality and morbidity due to the disease. Factors affecting early detection include individual determinants, social determinants and health system determinants. Individual determinants have been defined through the health belief model. In order to achieve the desired outcome it is necessary to modify the determinants resulting in the desired behaviour.

\section{The Health Belief Model}

The Health Belief Model was developed by social scientists to understand the reluctance or failure of people to adopt disease preventing strategies, screening tests and other health promoting behaviours. This was later expanded to understand peoples' response to symptoms and compliance to medical treatment.

The HBM derives from psychological and behavioural theory with the postulates that the two components of health-related behaviour are

1) The desire to avoid illness, or conversely get well if already ill;

2) The belief that a specific health action will prevent, or cure, illness.

An individual's course of action often and behaviour depends on the person's perceptions of the benefits and barriers related to health behaviour. There are six constructs of the HBM. The first four constructs were developed as the original postulates of the HBM. The last two were added as research about the HBM evolved.

1. Perceived susceptibility - This refers to a person's subjective perception of the risk of acquiring an illness or disease. There is wide variation in a person's feelings of personal vulnerability to an illness or disease.

2. Perceived severity - This refers to a person's feelings on the seriousness of contracting an illness or disease (or leaving the illness or disease untreated). There is wide variation in a person's feelings of severity, and often a person considers the medical consequences (e.g., death, disability) and social consequences (e.g., family life, social relationships) when evaluating the severity.

3. Perceived benefits - This refers to a person's perception of the effectiveness of various actions available to reduce the threat of illness or disease (or to cure illness or disease). The course of action a person takes in preventing (or curing) illness or disease relies on consideration and evaluation of both perceived susceptibility and perceived benefit, such that the person would accept the recommended health action if it was perceived as beneficial.

4. Perceived barriers - This refers to a person's feelings on the obstacles to performing a recommended health action. There is wide variation in a person's feelings of barriers, or impediments, which lead to a cost/benefit analysis. The person weighs the effectiveness of the actions against the perceptions that it may be expensive, dangerous (e.g., side effects), unpleasant (e.g., painful), time-consuming, or inconvenient.

5. Cue to action - This is the stimulus needed to trigger the decision-making process to accept a recommended health action. These cues can be internal (e.g., chest pains, wheezing, etc.) or external (e.g., advice from others, illness of family member, newspaper article, etc.).

6. Self-efficacy - This refers to the level of a person's confidence in his or her ability to successfully perform a 
behavior. This construct was added to the model most recently in mid-1980. Self-efficacy is a construct in many behavioural theories as it directly relates to whether a person performs the desired behavior.

\section{The Systems Approach}

The Systems Approach looks at the entire ecosystem in which all the determinants co-exist. While the health belief model addresses the individual and social determinants, this alone is not sufficient to achieve the desired outcome. The health system should be equipped with the infrastructure and man power such that a woman can undergo screening. The health system can be broadly bifurcated as public and private health care. The public system may be more affordable for the common man, however the quantum is less and therefore not easily accessible to all populations. Similarly, the private healthcare system may have more infrastructure and man power but this is not affordable to the common man.

Based on the conceptual framework an intervention was designed that aimed to decrease the mortality and morbidity of breast cancer by enabling early detection through screening services.

\section{The intervention}

Early in 2014, Narayana Health through its CSR initiative launched a Breast Cancer Screening and awareness program based on the framework discussed above. The program encompassed the following objectives

\section{- Creating awareness about breast cance}

- Screening for breast cancer

- Follow up and referral of detected patients to the nearest facility

\section{Awareness Creation}

The first component of the program is achieved by conducting an awareness session. The main aspect of the intervention is creating awareness about risk factors, protective factors and behaviours to be adopted. The women are also trained on how to conduct Self Breast Examination. Awareness creation is an important aspect in bringing about behavioural change with respect to Breast Cancer. The session is conducted by trained health workers and is structured based on the Health Belief model thus addressing the various individual and social determinants. Awareness sessions are conducted in a group, this encourages dialogue between women enabling a platform to discuss an issue they may consider sensitive. Stigmas, taboos and myths associated with breast cancer are busted and women are encouraged to take up the issue in their own circles becoming advocates. Described below is how the awareness session addresses each of the individual determinants:

1. Perceived susceptibility - In many areas breast cancer is seen as a very 'distant disease', something the people have heard about but have a false sense of confidence that it will not happen to them. A lot of women in rural areas perceive breast cancer to be a disease of the more affluent populations in developed regions. The awareness sessions present to the women statistics that are alarming. In addition, risk factors for the disease are also covered which serve as an eye opener. Women come to understand that they are not alien to many of the risk factors. This is the first step towards encouraging them to seek preventive/early detection procedures.

2. Perceived Severity - It is of utmost importance that each individual is aware of the physical, mental and financial implications of cancer. This is communicated in the awareness sessions through a combination of statistics pertaining to the cost of treatment of cancer. Huge emphasis is given on the fact that cancer detected at an early stage is better cured and the treatment costs less. The main point communicated that cancer despite being a grave disease, the severity is much less and survival rates better if detected at an early stage and hence the importance of undergoing periodic screening arises.

3. Perceived Benefits - The benefits of screening and early detection of breast cancer are linked to the severity. The principal benefit of screening is early detection. A lot of evidence exists that cancer detected at an early stage decreases the overall burden on the individual, their family/community and the entire health system at a large. A behaviour as simple as self-breast examination has an impact on several different levels.

4. Perceived Barriers - The major barriers to seeking breast cancer screening services are understood to be availability, accessibility and affordability. The program directly addresses this challenge and helps women from underprivileged and low resource settings to overcome these barriers by taking the screening services to them. The women receive comprehensive screening services, risk assessment and counselling at no cost. The stigma associated with the breast' or with breast cancer is a major barrier that exists. Women are generally uncomfortable discussing about their breasts or any changes they may have noticed or experienced. The awareness session targets to break this stigma or taboo faced by women whereby they are not able to open about changes in their body that affects their health. The sessions enable a conversation about what is considered a sensitive issue and busts several associated myths.

5. Cues to Action - The entire program is a cue to action. It empowers women with information about a disease they are susceptible to and enables them to get themselves screened. It is trusted that this action is extended unto their families and communities.

6. Self-efficacy - The program helps women understand the disease better and that it is not beyond their power to stay safe and healthy. Conducting screening alone does not ensure sustainability of the health promoting behaviour being advocated. The awareness session busts a lot of myths associated with breast cancer, addresses the stigma linked to breast cancer and provides evidence-based information on good practices and behaviours to reduce risk of breast cancer as well as enable early detection.

Following the awareness session, women are then screened for breast cancer by a doctor/trained healthcare worker.

\section{Breast Cancer Screening}

The screening component of the program addresses the health system determinants. Narayana Health was supported in the program by Nilekani Philanthropies. They donated a bus that was fully equipped with a breast examination room, waiting area and mammography unit. Narayana Health CSR was mandated with the effective and efficient utilisation of this asset banking of its expertise in the space of cancer surveillance and treatment. Senior oncologists from Narayana Health trained health workers to carry out clinical breast examination and to conduct awareness sessions on the deadly disease. It is beyond the scope of the program to entirely modify or influence the health system determinants, however the program does try to bridge the gap by providing mammography screening services for free to populations from a poor socio-economic status through the Mobile Mammography Unit.

\section{Protocol for Breast Cancer Screening}

The most commonly and highly recommended test for screening for breast cancer is mammography. This modality 
however is expensive and exposes the woman to low dose radiation. Narayana Health adopted the WHO recommended protocol for Breast Cancer Screening in low resource settings. All women undergo Clinical Breast Examination by a doctor or trained healthcare professional. Women who are suspected to have a lump/abnormality in the breast tissue are then advised for further tests. Women above the age of 40 years undergo Mammogram while women below 40 years of age undergo an Ultrasound scan for further confirmation. Mammography services are available at the camp location through the mobile mammography unit, however Ultrasound is not available. Women who were suggested to undergo an ultrasound are then followed up via call and encouraged to visit their nearest healthcare facility and undergo the test. All women are trained on conducting Self Breast Examination and all women aged above 40 years are encouraged to get a mammogram at least once in every 2 years.

The program aims to be cost effective while maintaining quality of the tests performed. In order for this, qualified staff are selected and given intensive training to carry out clinical breast examination. The Mobile Mammography Unit is staffed with a Mammography Technician, and a nursing staff who has been trained on clinical breast examination and history taking. The bus has been designed such that there is a waiting area, a room with a bed to perform clinical breast examination and a room that houses the mammography machine. The bus visits low resource settings in rural areas and urban slums where the populations do not have access to healthcare services or are unable to afford the same. Women aged above 40 years who are suspected undergo free mammography in the MMU. Periodic follow up is done to ensure that women who were identified as high risk/suspected seek and receive treatment.

\section{Framework}

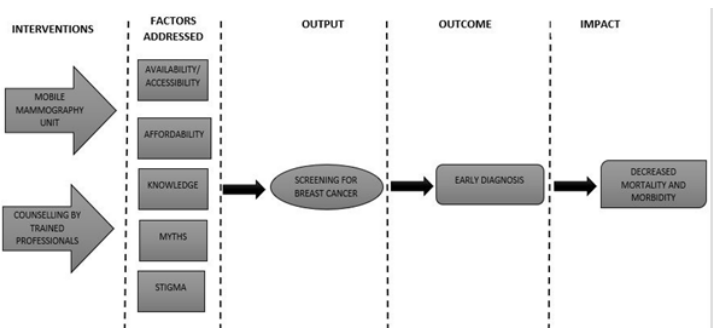

Results

A total of 30408 women were screened for breast cancer since 2015 through 757 camps across Bangalore, Mysore, Shimoga and Bellary. Of the total women screened 7200 women underwent mammography at the bus and 1964 were referred to the oncologist. 47 cases of breast cancer were identified in the early stages.

\begin{tabular}{|c|c|}
\hline Particular & Number \\
\hline Total Number of Camps & 757 \\
\hline $\begin{array}{c}\text { Total number of awareness } \\
\text { sessions conducted }\end{array}$ & 563 \\
\hline Total Women & 30408 \\
\hline Total Mammograms & 7200 \\
\hline $\begin{array}{c}\text { Total suspected cases referred } \\
\text { to oncologist }\end{array}$ & 1964 \\
\hline Breast cancer cases identified & 47 \\
\hline
\end{tabular}

\section{Key challenges}

Like every public health initiative, this too has faced several challenges in achieving the objectives. The challenges faced are internal, operational as well as societal:

\section{Internal Challenges}

Internal challenges refers to challenges faced within the core program team. As has been discussed in the case study, the program focuses on training health workers rather than depending on professionals such as doctors or nurses. Retention and motivation of these health workers is a huge challenge. They can be trained on skills such as clinical breast examination or history taken, however certain intricacies are learnt only with field experience which takes time. For example, despite being guided that there are no targets to be achieved due to conventional work place practices the health worker sometimes feels that they have to present cases to prove they are working. This can lead to reporting of false positives. A better understanding of public health is required to eliminate this self-embraced pressure from the health workers.

\section{Operational Challenges}

The program involves several verticals working together (biomedical, information technology, radiology, maintenance, administration). Co-ordination between all the departments is key to efficient functioning and timely output. This sometimes proves to be a challenge for the program.

\section{Societal and Systemic Challenges}

Reaching out to different strata in society and addressing cultural barriers that are preventing women from coming forward is one of the main objectives of the program. The modus operandi followed in the program involves collaborating with organisations, both public as well as private that work at the grass root levels. This helps us to reach a huge population but also limits us. We are unable to make available the service to sects of the community that have not been reached by other grass root organisations. The program itself does not have the bandwidth to go out and create a connect with the community, dependency lies on already established institutions and organisations.

The team functions with minimal resources, trained healthcare workers are the primary resource. Even though they are well trained by doctors, it is sometimes difficult to get acceptance from the community. Society has been conditioned to trust only a doctor advise when it comes to healthcare. However, with the crunch in trained doctors and specialists it becomes necessary to bank on existing human resource and train the sufficiently that they may disseminate the required information in a desired manner.

Loss to follow up is a significant challenge faced. Any screening program is successful only when the loop is closed i.e. early detection should be followed by seeking treatment/prevention. Otherwise, the screening activity does not contribute to a decrease in mortality/morbidity. Once a respondent screened is suspected to be suffering from breast cancer it is essential that they undergo the confirmatory diagnostic tests and initiate treatment. Many women do not have a phone number, or the number provided is not reachable, thus is becomes difficult to follow up with them.

The women who attend screening camps are usually from a lower socio-economic stratum, any suspected cases are encouraged to seek further treatment at nearby government institutions. Unfortunately, public health facilities equipped to treat/diagnose cancer are not available at a rural setting. This is a challenge that must be addressed by the public health system at large.

Ideally every woman must be screened for breast cancer, practically more efficient use of resources would be achieved if women who have a high risk or women residing in geographical settings that put them at higher risk are screened on priority. Unfortunately, there is a scarcity of such data that could improve the impact of the program several folds.

Recommendations

The framework for the program broadly describes factors that 
influence women taking up a desired health behaviour. The program took on certain interventions to address these factors and through field experience it was understood that there are more approaches that can be used to get the desired output. For example, individual determinants are being addressed through awareness sessions. On engaging with the women on field we have understood that lack of awareness alone is not a reason, an extra motivation or reinforcement would go a long way to encourage the desired behaviour (Nudge Theory). Our experience on working with the individual determinants has led us to understand that the Nudge theory is of great importance. This can be taken forward through mass messaging of public health matters. Women could get a reminder SMS asking them to go get screened or following up with them. Also more influential 'nudges' for example from a religious leader, a film actor/actress, a sports star etc could prove to be more encouraging to the women. This can be piloted to better understand the scope of including 'nudge' as an intervention in order to improve the outcomes and impact of the program.

Sharing of survivor stories is an approach that has an emotional touch. Women can hear stories from breast cancer survivors who come from similar walks of life. This would help them develop a better connect and understanding about the disease and the journey of fighting the disease. The sharing could be done by developing videos or live sessions by survivors at the camp locations based on their availability and level of comfort. Testimonials delivered by survivors are without doubt one of the most powerful and persuasive messages.

As already discussed, while the program aims to ensure all women get screened for breast cancer, it is also of utmost importance that high-risk women are able to access this on a priority basis. The Ministry of Health and Family Welfare as part of its Comprehensive Primary Health Care introduced guidelines for Prevention Screening and Control of NonCommunicable Diseases. As part of this the ASHA Workers are required to fill in a Community Based Risk Assessment format (CBAC) which helps identify the high-risk women. Assuming that this is happening, collaboration with the National Health Mission and access to this information would ensure that the high-risk cohort benefits from the initiative from Narayana Health. Such a collaboration would better address the health system determinants.

Narayana Health through its CSR initiatives aspires to reach out to the lesser privileged in a most efficient manner and greatly values any partnerships or collaborations that would help it to do so.

\section{REFERENCES}

1. Agarwal, G., \& Ramakant, P. (2008). Breast Cancer Care in India:The current Scenario and Challenges fr the Future. Breast Care, Karger.

2. BO, A., CH, Y., Smith, R., R, S., SF, S., \& A, E. (2008). Guideline implementation for breast healthcare in low-income and middle-income countries: overview of the Breast Health Global Initiative Global . Cancer, 2221-2243.

3. Ferlay, J., Colombet, M., Soerjomataram, I., Mathers, C., Parkin, D., Pineros, M., \& Znaor, A. (2018). Estimating the global cancer incidence and mortality in 2018: GLOBOCAN sources and methods. International Journal of Cancer.

4. Ferlay, J., Dikshit, R., \& Soerjomataram, I. (2015). Cancer incidence and mortality worldwide: sources, methods and major patterns in GLOBOCAN 2012. International Journal of Cancer.

5. Ferlay, J., Shin, H., Bray, F., Forman, D., Mathers, C., \& Parkin , D. (2008). Estimates of worldwide burden of Caancer in 2008: GLOBOCAN 2008. International Journal of Cancer, 2893-917.

6. Gajalakshmi, V., Mathew, A., \& Brennan, P. (2009). Breastfeeding and breast cancer risk in India: a multicenter case-control study. International Journal of Cancer.

7. ICMR. (2014). National Cancer Registry Program Annual Report. Indian Council of Medical Research.

8. K.A., D., Shastri, S., \& Patil, S. (2010). Cancer Control Program in India; Challenges for the New Millenium. Health Administrator.

9. Madhav, M. R., Nayagam, S. G., Biyani, K., \& Pandey, V. (2018). Epidemiologic analysis of breast cancer incidence, prevalence and mortaity in India. Medicine.

10. Malvia, S., Dubey, U., Bagadi, S. A., \& Saxena, S. (2017). Epidemiology of breast cancer in Indian women. Asia Pacific Journal of Clinical Oncology.
11. Pakseresht, S., Ingle, G., \& Bahadur, A. (2009). Risk factors with breast cancer among women in Delhi. Indian Journal of Cancer.

12. Shrivastava, S., Shrivastava, P., \& Ramasamy, J. (2013). Self Breast Examination: A tool for early diagnosis of Breast Cancer. American Journal of Public Health.

13. Singh, S., \& Shrivastava, P. J. (2015). Breast cancer screening existence in India: A nonexisting reality. Indian Journal of Medical and Paediatric Oncology.

14. Surakasula, A., Nagarjunapu, G., \& Ragavaiah, K. (2014). A comparative study of pre and post menopausal breast cancer: risk factors, presentation, characteristics and management. Journal of Research in Pharmacy Practice. 